\title{
HACIA EL DESARROLLO SOSTENIBLE DE ORGANIZACIONES Y EMPRESAS: LA HUELLA ECOLÓGICA CORPORATIVA Y SU APLICACIÓN A UN PRODUCTOR DE MEJILLÓN EN GALICIA (ESPAÑA)
}

\author{
Adolfo Carballo Penela, María do Carme García Negro \\ Universidad de Santiago de Compostela \\ Departamento de Economía Aplicada.
}

"Grupo de Investigación de Economía Pesquera y Recursos Naturales Av. Burgo das Nacións s/n. Santiago de Compostela. CP. 15782. A Coruña, Galicia-España. e-mail: adolfo.carballo@usc.es

Manizales, 2008-10-06 (Rev. 2008-11-30)

\section{RESUMEN}

La Huella Ecológica (HE) es un indicador que proporciona un marco de análisis para el estudio de la demanda humana de bioproductividad. En los últimos años se ha avanzado en su aplicación a organizaciones, destacándose su potencialidad como herramienta de gestión empresarial. Este artículo parte de la exposición de cuestiones generales relacionadas con el funcionamiento del indicador. Seguidamente, se señalan las diferentes opciones de cálculo en lo que se refiere a la huella de empresas, estimando la HE de una empresa productora de mejillón en dos años diferentes, 2002 y 2007, de acuerdo al método compuesto de las cuentas contables (MC3). El objetivo del estudio es detectar qué actividades realizadas por la empresa generan una mayor huella y, además, analizar la evolución del indicador en el período considerado. Los resultados muestran una HE reducida, causada principalmente por el consumo de combustibles de la embarcación de la empresa. Así mismo, se produce un incremento de la huella en 2007, debido a dos hechos puntuales relacionados con la sustitución de una batea y reparaciones en la embarcación.

\section{PALABRAS CLAVE}

Sostenibilidad, huella ecológica corporativa, mejillón.

\section{TOWARDS SUSTAINABLE DEVELOPMENT IN ORGANIZATIONS AND BUSINESSES: THE CORPORATIVE ECOLOGICAL FOOTPRINT AND ITS APPLICATION TO A MUSSEL PRODUCER IN GALICIA, SPAIN}

\begin{abstract}
The ecological footprint (EF) is an indicator which provides a referential framework for the analysis of human demand of bioproductivity. In recent years, it has been applied to organizations, highlighting its potential as a business management tool. Firstly, this article introduces general questions regarding the indicator's functionality. Secondly, corporate calculation methods are presented. Finally, the EF of a mussel producer company in two different years, 2002 and 2007, was estimated using the "compound method of financial accounting" (MC3). This study aims to find out which activities carried out by the company generate a higher
\end{abstract}


footprint, as well as analyzing the evolution of the indicator in the study period. Results show a reduced footprint, basically related with fuel consumption of, used in the vessel that the company owns. There is also an increase in the footprint in 2007, due to concrete facts related to raft replacement and vessel repair.

\section{KEY WORDS}

Sustainability, corporate ecological footprint, mussel.

\section{AGRADECIMIENTOS}

Queremos agradecer la colaboración de Antonio Recuna Cuiña, representante de la empresa Alfa, sin cuya colaboración no habría sido posible este trabajo.

\section{LA HUELLA ECOLÓGICA}

La huella ecológica (HE) es un indicador de sostenibilidad desarrollado a principios de los 90 por William Rees y Mathis Wackernagel. El concepto relaciona las necesidades de capital natural de una economía, con el área biológicamente productiva que le corresponde (Wackernagel et al., 1999), siendo diseñado como una herramienta de planificación para medir la sostenibilidad ecológica (Wackernagel et al., 1999; Wackernagel y Silverstein, 2000) con el propósito de estimar la magnitud del consumo humano que excede la capacidad de regeneración de la biosfera (Wackernagel, 1999).

La HE es definida como "La superficie de tierra productiva o ecosistema acuático necesario para mantener el consumo de recursos y energía, así como para absorber los residuos producidos por una determinada población humana o economía, considerando la tecnología existente, independientemente de en qué parte del planeta está situada esa superficie"1. Su punto de partida es la asunción de que, tanto el consumo de recursos, como la generación de residuos, pueden ser convertidos en la superficie productiva necesaria para mantener estos niveles de consumo o, en otros términos, en la Huella Ecológica. Ésta es comparada con la superficie disponible, asumiendo que las poblaciones con una huella superior a la superficie de la que disponen son insostenibles (Lenzen et al., 2003), existiendo lo que se denomina déficit ecológico ${ }^{2}$.

La HE es dividida en distintas subhuellas. En la mayoría de los estudios realizados se emplean las seis siguientes:

- Cultivos: aquella superficie en la que los humanos desarrollan actividades agrícolas, suministrando productos como alimentos, fibra, aceites, etc.

- Pastos: área dedicada a pastos, de donde se obtienen determinados productos animales como carne, leche, cueros y lana.

- Bosques: la superficie ocupada por los bosques, de donde principalmente se obtienen productos derivados de la madera, empleados en la producción de bienes, o también de combustibles como leña.

- Mar: la superficie marítima biológicamente productiva aprovechada por los humanos para obtener pescado y mariscos.

- Superficie construida: área ocupada por edificios, embalses, carreteras y otras infraestructuras, etc., por lo que no es biológicamente productiva. 
- Energía: el área de bosque necesaria para absorber las emisiones de $\mathrm{CO}_{2}$ procedentes de la quema de combustibles fósiles.

Igualmente, suelen distinguirse distintas categorías de consumo, de modo que, para cada una de ellas, se establecen las distintas necesidades de superficie. Concretamente, la propuesta inicial de los autores establece 5 categorías de consumo: alimentación, hogar, transporte, bienes de consumo, servicios, que a su vez pueden ser divididas en las subcategorías que se consideren oportunas.

Tabla 1. Matriz de superficies apropiadas por categoría de consumo (Ha/cápita)

\begin{tabular}{|l|l|l|l|l|l|l|l|}
\hline & $\begin{array}{c}\text { Cultiv } \\
\text { os }\end{array}$ & $\begin{array}{c}\text { Past } \\
\text { os }\end{array}$ & $\begin{array}{c}\text { Bosqu } \\
\text { es }\end{array}$ & $\begin{array}{c}\text { Superfi } \\
\text { cie } \\
\text { construi } \\
\text { da }\end{array}$ & $\begin{array}{c}\text { Energ } \\
\text { ía }\end{array}$ & $\begin{array}{c}\text { M } \\
\text { ar }\end{array}$ & $\begin{array}{c}\text { Tot } \\
\text { al }\end{array}$ \\
\hline $\begin{array}{l}\text { Alimentaci } \\
\text { ón }\end{array}$ & & & & & & & \\
\hline Hogar & & & & & & & \\
\hline Transporte & & & & & & & \\
\hline $\begin{array}{l}\text { Bienes de } \\
\text { consumo }\end{array}$ & & & & & & & \\
\hline Servicios & & & & & & & \\
\hline TOTAL & & & & & & & $\begin{array}{l}\text { HE } \\
\text { TOT } \\
\text { AL }\end{array}$ \\
\hline
\end{tabular}

Fuente: Elaboración propia a partir de Wackernagel y Rees (1996)

Uniendo las categorías de consumo con las categorías del uso de la Tierra, se obtiene una matriz consumo-superficies, donde cada fila muestra la apropiación de superficie de una categoría de consumo concreta, y cada columna la distribución de la apropiación de un tipo de superficie entre los distintos tipos de consumo.

\section{MÉTODO DE CÁLCULO}

El método de cálculo propuesto por Wackernagel y colegas, denominado método compuesto, implica el empleo de estadísticas de consumo y población, con la finalidad de estimar el consumo anual per cápita ${ }^{3}$. Se comienza determinando el consumo total de cada producto por los habitantes del territorio estudiado. Esta labor se suele realizar de un modo indirecto, añadiendo a la producción de cada bien las cantidades importadas, restando las exportaciones del mismo. Una vez hecho esto, se divide el consumo total por la población, obteniendo un valor medio por habitante (t/hab.).

Una vez calculado el consumo de cada categoría de producto por habitante, debemos transformarlo en la superficie necesaria para producir la cantidad consumida. Para esta labor, el método propuesto emplea información de la productividad biológica de la superficie de donde se obtiene el producto en cuestión, expresada en toneladas por hectárea (t/ha). Así, dividiendo el consumo ( $t / h a b$.) entre el rendimiento ( $t / h a)$, obtenemos las hectáreas de superficie que cada habitante necesita para obtener la cantidad consumida de cada categoría ${ }^{4}$. 


\subsection{LA HUELLA DE LA ENERGÍA}

El cálculo de la HE de la energía reviste ciertas particularidades, pues la relación consumo-productividad no es fácilmente visible. En relación al consumo de combustibles fósiles, el método compuesto parte de estimar las emisiones de $\mathrm{CO}_{2}$ generadas en la economía estudiada, tratando de determinar la superficie de los bosques necesaria para absorber las emisiones. Para eso, hace falta determinar una tasa representativa de la cantidad de $\mathrm{CO}_{2}$ que puede absorber una hectárea de bosque, aplicando inicialmente -Wackernagel y Rees (1996)- una tasa de absorción de 6,6 $\mathrm{tCO}_{2} /$ ha/año, obtenida de estudios referidos a los bosques canadienses. Otro valor comúnmente empleado son los $5,21 \mathrm{tCO}_{2} /$ ha/año que propone el Panel internacional sobre Cambio Climático (IPCC, 2001).

En el caso de la energía nuclear, es difícil calcular su HE por diversos motivos. La alta densidad de los combustibles empleados en las centrales nucleares, propicia que la demanda de productividad biológica sea muy baja en relación a la cantidad de energía producida (Wackernagel y Monfreda, 2004). Igualmente, la capacidad de la biosfera para asimilar materiales radiactivos es mínima, y los efectos devastadores en el territorio en caso de accidente, incrementarían sustancialmente la huella ecológica de esta fuente de energía ${ }^{5}$.

No obstante, se considera que su exclusión de la HE podría ser interpretada en el sentido de que los países donde la energía nuclear tiene una fuerte presencia son más sostenibles (Monfreda et al, 2004). De ahí que se estime su huella considerando que la energía nuclear consumida es obtenida de un combustible fósil, a pesar de que, tal y como señalan Mc Donald y Patterson, (2003), la energía nuclear y los combustibles fósiles tienen impactos ambientales sustancialmente diferentes.

En cuanto a las energías renovables, la HE de este tipo de energías incluye tanto la superficie ocupada por las instalaciones empleadas para producir electricidad (la superficie inundada por las presas, los aerogeneradores y los paneles solares ${ }^{6}$ ), la energía incorporada en estas infraestructuras, como la pérdida de productividad debido al uso de esta superficie para la producción de energía. Finalmente, hace falta destacar la necesidad de considerar la energía incorporada a los flujos de comercio exterior, descontando de la HE de cada país la cantidad de energía incorporada a los bienes que se exportan e incorporando la energía producida en otras economías, pero consumida por los habitantes del territorio estudiado mediante la importación de bienes ${ }^{7}$.

\section{LA HUELLA ECOLÓGICA CORPORATIVA (HEC)}

\subsection{Concepto y objetivos}

Tanto porque la legislación ambiental está cada vez más desarrollada como por la presión de consumidores y la propia concienciación de los dirigentes, las empresas deben asumir cada vez compromisos ambientales mayores, contribuyendo al logro de un medio ambiente sostenible o, cuando menos, evitando su degradación. En este contexto, resulta interesante la elaboración de una herramienta que muestre, de modo simple, la situación medioambiental de organizaciones y empresas, debiendo ser un elemento útil para la toma de decisiones al respecto. Surge, entonces, la posibilidad de emplear la HE con este fin, pues es un indicador que sintetiza diferentes efectos en el medio ambiente; su cálculo es relativamente 
simple; se expresa en unidades fácilmente comprensibles siendo, por tanto, útil para la toma de decisiones.

La aplicación de la HE a organizaciones era una posibilidad señalada por los propios creadores del indicador (Wackernagel y Rees, 1996), pues dado que las empresas al igual que los ciudadanos son consumidoras de recursos y generadoras de residuos, tenemos los elementos necesarios para calcular una huella.

En su contra, podría cuestionarse el sentido de que las empresas empleen un indicador expresado en una unidad, las hectáreas de superficie productiva, apropiado para países o regiones, pero quizás, menos relacionado con corporaciones. No obstante, tal y como justificaremos en la sección siguiente, la superficie ocupada puede tener también sentido en una HEC. Por otro lado, las diferentes metodologías que se vienen aplicando permiten expresar la HEC no sólo en términos de hectáreas, sino en términos de emisiones, principalmente de $\mathrm{CO}_{2}$, surgiendo lo que algunos autores denominan huella del carbono ${ }^{8}$. Esta adaptación es notablemente atractiva para las empresas, sobre todo considerando las exigencias que deben afrontar en el marco del Protocolo de Kyoto.

Igualmente, la HEC puede ser también relacionada con los bienes que cada empresa produce. Su consideración en términos de hectáreas o toneladas de $\mathrm{CO}_{2}$, por tonelada de producto, confiere al indicador la capacidad de ser empleado como elemento en una ecoetiqueta (Doménech, 2007). De este modo, el consumidor dispondría de información medioambiental relevante y comprensible al respecto de los bienes que consume y de las empresas productoras.

\subsection{Método de cálculo}

Al igual que sucede con la huella en su versión tradicional, no existe un único método de cálculo para empresas y organizaciones. Es más, partiendo de la metodología general se pueden calcular, sin necesidad de muchos cambios, huellas de determinados consumos, existiendo incertidumbre al respecto de qué conceptos se incluyen en el cálculo de la HEC. Por otro lado, ni Wackernagel ni Rees han propuesto, por el momento, una metodología específica para las organizaciones, por lo que no existe una referencia clara, como en el caso de países y regiones ${ }^{9}$.

\subsubsection{Descripción de la metodología seleccionada}

En nuestro estudio, hemos empleado el "método compuesto de las cuentas contables" (MC3), desarrollado por Doménech (2004a, 2004b, 2007). El origen del MC3, podemos encontrarlo en la huella familiar (Wackernagel et. al., 2000). Basándose en la matriz de consumos y superficies presente en la hoja de cálculo elaborada para el cálculo de la huella de los hogares, Doménech (2004a, 2004b, 2007) elabora una matriz que recoge los consumos de las principales categorías de productos que una empresa necesita. Su referencia en este trabajo fue la Autoridad Portuaria de Gijón, la primera organización a la que se aplicó esta metodología.

La idea general es elaborar un listado de las principales categorías de productos consumidos por una empresa, existiendo también apartados para los residuos generados y el uso del suelo. Tal y como señalamos a continuación, la huella se expresa tanto en hectáreas como en toneladas de $\mathrm{CO}_{2}{ }^{10}$. 
La información necesaria se obtiene, principalmente, de documentos contables como el balance y la cuenta de pérdidas y ganancias, bien puede ser necesaria información de otros departamentos de la empresa que dispongan de información específica de determinados apartados (generación de residuos, superficie ocupada por las instalaciones de la organización, etc.).

\section{La matriz de consumos-superficies}

Esta matriz se implementa en una hoja de cálculo, la principal herramienta para el cálculo de la HEC. Su estructura no difiere, en lo sustancial, de la matriz de consumos-superficies empleada en el cálculo de la HE de países. Así, las filas muestran la huella de cada categoría de producto, mientras que las columnas de la matriz también incluyen, entre otras cosas, las distintas superficies en las que se divide la huella. Sin embargo, la hoja de cálculo elaborada para estimar la HEC es más compleja, tal y como describimos a continuación (Ver la Tabla 2).

Tabla 2. Estructura de la hoja de cálculo que recoge la matriz consumos-superficies de la HEC

\begin{tabular}{|c|c|c|c|c|c|c|c|}
\hline \multirow[b]{2}{*}{$\begin{array}{l}\text { CATEGORIAA DE } \\
\text { PRODUCTO }\end{array}$} & \multicolumn{5}{|c|}{ ConSumo anual } & \multicolumn{2}{|c|}{ Productividad } \\
\hline & 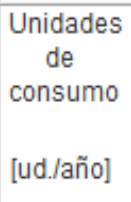 & $\begin{array}{l}\text { Euros sln } \\
\text { IVA } \\
\text { [euros/año] }\end{array}$ & $\begin{array}{c}\text { Toneladas } \\
\text { [t/año] }\end{array}$ & $\begin{array}{c}\text { Intensidad } \\
\text { energética } \\
\text { [Gj/t] }\end{array}$ & $\begin{array}{c}\text { Gj } \\
\text { [Gj/año] }\end{array}$ & $\begin{array}{l}\text { Natural } \\
\text { [tha/año] }\end{array}$ & $\begin{array}{l}\text { Energética } \\
\text { [Gj/ha/año] }\end{array}$ \\
\hline 1. ENERGÍA & & & & & & & \\
\hline 1.1 Electricidad & & & & & & & \\
\hline 1.2 Combustibles & & & & & & & \\
\hline 1.3 Materiales & & & & & & & \\
\hline $\begin{array}{l}1.4 \text { Materiales de } \\
\text { construcción }\end{array}$ & & & & & & & \\
\hline 1.5 Servicios & & & & & & & \\
\hline 1.6 Residuos y vertic & & & & & & & \\
\hline 2. USO DEL SUELO & & & & & & & \\
\hline $\begin{array}{l}\text { 3. RECURSOS } \\
\text { AGROPECUARIOS Y } \\
\text { PESQUEROS }\end{array}$ & & & & & & & \\
\hline $\begin{array}{l}\text { 4. RECURSOS } \\
\text { FORESTALES }\end{array}$ & & & & & & & \\
\hline
\end{tabular}


Tabla 2. Estructura de la hoja de cálculo que recoge la matriz consumos-superficies de la HEC (continuación)

\begin{tabular}{|c|c|c|c|c|c|c|c|c|}
\hline \multirow[b]{2}{*}{$\begin{array}{l}\text { CATEGORIAA DE } \\
\text { PRODUCTO }\end{array}$} & \multicolumn{6}{|c|}{ HUELLA por tipo de ecosistema } & \multirow[b]{2}{*}{$\begin{array}{c}\text { HEC } \\
\text { TOTAL } \\
\text { [Ha*FE] }\end{array}$} & \multirow{2}{*}{$\begin{array}{c}\text { CONTRAHUE } \\
\text {-LLA } \\
\text { [Ha*FE*FR] }\end{array}$} \\
\hline & \begin{tabular}{|l|} 
Energía \\
Fósil \\
{$\left[\mathrm{Ha} a^{\star} \mathrm{FE}\right]$}
\end{tabular} & $\begin{array}{l}\text { Tierra } \\
\text { Cultivable } \\
\text { [Ha*FE] }\end{array}$ & $\begin{array}{l}\text { Pastos } \\
{\left[\mathrm{Ha} a^{\star} \mathrm{FE}\right]}\end{array}$ & \begin{tabular}{|l|} 
Bosques \\
{$\left[\mathrm{Ha} \mathrm{F}^{\star} \mathrm{FE}\right]$}
\end{tabular} & $\begin{array}{l}\text { Terreno } \\
\text { Construido } \\
\text { [Ha*FE] } \\
\end{array}$ & $\begin{array}{c}\text { Mar } \\
{\left[\mathrm{Ha}{ }^{\star} \mathrm{FE}\right]}\end{array}$ & & \\
\hline 1. ENERGIA & & & & & & & & \\
\hline 1.1 Electricidad & & & & & & & & \\
\hline 1.2 Combustibles & & & & & & & & \\
\hline 1.3 Materiales & & & & & & & & \\
\hline $\begin{array}{l}1.4 \text { Materiales de } \\
\text { construcción }\end{array}$ & & & & & & & & \\
\hline 1.5 Servicios & & & & & & & & \\
\hline $\begin{array}{l}\text { 1.6 Residuos y } \\
\text { vertidos }\end{array}$ & & & & & & & & \\
\hline $\begin{array}{l}\text { 2. USO DEL } \\
\text { SUELO }\end{array}$ & & & & & & & & \\
\hline $\begin{array}{l}\text { 3. RECURSOS } \\
\text { AGROPECUARIOS } \\
\text { Y PESQUEROS }\end{array}$ & & & & & & & & \\
\hline $\begin{array}{l}\text { 4. RECURSOS } \\
\text { FORESTALES }\end{array}$ & & & & & & & & \\
\hline
\end{tabular}

Comenzando por las columnas, existen en 6 grupos, correspondiendo el primero (columna 1) a la descripción de las diferentes categorías de productos consumibles. Estos están agrupados en cuatro grandes bloques: consumo energético, a su vez distribuido en seis subgrupos ${ }^{11}$, uso del suelo, recursos agropecuarios y pesqueros y recursos forestales. Dentro de cada grupo se pueden incluir tantos productos como se desee.

El segundo grupo (columnas 2-6) muestra los consumos de cada producto, expresados en unidades específicas. Las unidades de la primera columna del grupo se relacionan con las características del producto y, por ejemplo, el consumo de electricidad se recoge en $\mathrm{kwh}$, el de agua en $\mathrm{m}^{3}$, etc. La segunda columna recoge el valor de los consumos en euros, mientras que la tercera los muestra en toneladas. La quinta columna recoge la energía en gigajulios ( $\mathrm{Gj}$ ) de cada consumo, obtenida multiplicando las toneladas de producto por la cantidad de energía por tonelada empleada en su producción ( $\mathrm{Gj} / \mathrm{t})$, denominada intensidad energética, presente en la cuarta columna del grupo.

El tercer grupo de columnas (columnas 7-8) muestra la productividad de cada bien, expresada en dos columnas, la productividad natural, en toneladas por hectárea, y la productividad energética, en gigajulios por hectárea.

El cuarto grupo consta de seis columnas (9-14) que muestran la huella, repartida entre las diferentes superficies en las que se divide la huella, las mismas que en la huella de los países (energía fósil, tierra cultivable, pastos, bosques, superficie construida y mar).

Existe un último grupo (columnas 15-16) que recogen la huella ecológica total o el terreno ocupado y la contrahuella, o el terreno disponible. El concepto de contrahuella se describirá en apartados posteriores.

\section{Proceso de cálculo}


Una vez vista la estructura de la hoja de cálculo, podemos comenzar a ver cómo se calcula la HEC, lo que al mismo tiempo nos servirá para entender el significado de algunas de las nuevas columnas no presentes en la matriz tradicional de consumos-superficie. Nuestro objetivo no es describir detalladamente el método de cálculo, sino hacer una descripción general que nos permita comprender las principales ideas implícitas en él.

La metodología desarrollada por Doménech va a mantener la filosofía de la HE tradicional, con la particularidad de que una buena parte de los bienes que se consumen en una empresa no se obtienen de ninguna superficie productiva. Las empresas compran máquinas, ordenadores, consumen electricidad, todos ellos son bienes para los que la huella no puede ser calculada por el método tradicional, dividiendo el consumo entre la productividad de la superficie de la que proviene porque, al no ser bióticos, no proceden directamente de ninguna superficie.

Surge entonces un problema, pues la mayoría de consumos no se pueden computar en la huella de acuerdo a como se venía haciendo. En este caso se sigue, en cierto modo, el mismo razonamiento que en el caso de los países, pues el indicador recoge el impacto de la energía empleada en su producción. Sin embargo, el modo de estimar esta huella es ahora diferente.

En el caso de los territorios se tiene en cuenta el consumo de energía total de los habitantes del país o región estudiado, realizando un ajuste en función de las importaciones y exportaciones de bienes. Como en las empresas eso no es posible, Doménech (2004a, 2004b, 2007), se recurre a factores de intensidad energética que indican la energía consumida en la producción de cada categoría de producto, expresada en gigajulios por tonelada ${ }^{12}$. Tienen sentido, entonces, la cuarta y la quinta columna del segundo grupo de la hoja de cálculo, pues la energía total incorporada en la producción de cada producto se obtiene multiplicando el consumo en toneladas por la intensidad energética. En el caso de los bienes amortizables, la HE recoge cada año su cuota de amortización, evitando fluctuaciones elevadas en los períodos en los que se adquiera el inmovilizado.

Una vez que se obtiene la energía, se compara con la productividad energética de cada combustible, calculando de este modo la huella de los recursos no bióticos. La productividad energética se refiere a la cantidad de energía que puede asimilar una hectárea de bosque (Doménech, 2007), estimada a partir de la cantidad de energía contenida en las emisiones de $\mathrm{CO}_{2}$. Considerando que, para cada combustible existen factores estándar que recogen la cantidad de $\mathrm{CO}_{2}$ que emiten por Gj consumido, es posible realizar la operación inversa, estimando los Gj de cada combustible que fueron necesarios para emitir la cantidad $\mathrm{CO}_{2}$ que puede absorber anualmente una hectárea. En este sentido, la tasa de absorción de $\mathrm{CO}_{2}$ por hectárea empleada por Doménech no son las $6,6 \mathrm{tCO}_{2} /$ ha/año propuestas por Wackernagel y Rees (1996), sino que se actualiza este valor a 5,21 tCO $/ 2 /$ ha/año, de acuerdo a la propuesta del Panel Internacional sobre Cambio Climático (IPCC, 2001) ${ }^{13}$.

La estimación de la huella de la energía se aplica también a los servicios que contrata la organización estudiada y a los residuos que genera, ambos importantes en la huella de las organizaciones. En relación a los primeros, se asume que una parte del coste del servicio se corresponde al consumo energético, estimando el peso de esta parte para cada tipo de servicio. Este porcentaje se aplica al importe del servicio, obteniendo "los euros que se corresponden con el consumo energético" (Doménech, 2007, 96). Este valor se transforma en toneladas 
considerando el precio de los combustibles, procediendo luego a aplicar la intensidad energética correspondiente, del mismo modo que se haría al estimar la huella de la energía de cualquier otro recurso no biótico.

En lo tocante a los residuos, se estima la huella de la energía, con la particularidad de que se descuenta la cantidad de energía que se recupera en el proceso de reciclado, en el supuesto de que una parte de los residuos generados se reciclen.

Igualmente, una empresa también puede consumir recursos bióticos, como alimentos, madera, etc. En este caso, la estimación de la HEC se realiza por el método habitual de dividir el consumo, en este caso en toneladas, por la productividad natural de la superficie a la que se asigna cada producto ${ }^{14}$. No obstante, la huella de los recursos bióticos, debe incluir también la energía que se emplea en la producción de estos bienes, por lo que, además de la huella "natural o biótica", se estima su huella "energética", de igual modo que se haría para cualquier otro producto. Por lo tanto, la huella de este tipo de bienes incluye los dos componentes señalados, lo "natural" y lo "energético".

En relación con las productividades empleadas es necesario aclarar que se opta por el uso de productividades globales o mundiales pues, tal y como indica Doménech $(2007,84)$, "en el creciente mundo globalizado en el que vivimos, los artículos consumidos por las empresas o corporaciones tienen diversas procedencias, lo que haría muy dificultoso trabajar con las productividades locales de cada uno". Autores como Relea y Prat (1998) defienden esta opción para la huella de los países, afirmando que el uso de productividades locales puede provocar que aquellos con más recursos económicos consuman recursos de los lugares más productivos del planeta para reducir su huella vía productividad, dejando los productos de tierras menos productivas $\mathrm{y}$, por tanto, con mayor huella, a los países pobres.

Finalmente, en las organizaciones se recoge el uso del suelo, tanto en tierra firme como en el mar. En este caso, se diferencian distintos tipos de suelo (construido, zonas de cultivos, pastos), estimándose también la contrahuella de la organización.

La contrahuella es un concepto asimilable, en parte, a la superficie biológicamente productiva (SBP) de un país o región. Recordemos que en la versión clásica de la huella ecológica se compara la superficie necesaria para satisfacer las necesidades de los habitantes de un determinado territorio, la propia huella ecológica, con la superficie disponible para satisfacer esas necesidades, surgiendo un déficit o una reserva ecológica, dependiendo de cuál de las dos superficies fuese mayor.

Por ello, el concepto de SBP tiene sentido al hablar de territorios, pero no tanto en el caso de organizaciones. Todos los países disponen, en mayor o menor medida, de una parte de su superficie que es empleada para producir recursos bióticos, por lo que la comparación entre la superficie disponible y la consumida siempre va a ser posible. La huella ecológica asume que es positivo disponer de superficie productiva y, por tanto, que los habitantes del territorio estudiado satisfagan sus necesidades con productos producidos en el propio territorio. Desde el punto de vista de la condición de sostenibilidad, un país que no disponga de SBP nunca va a poder ser sostenible, pues sus habitantes deben consumir aunque sea simplemente para satisfacer sus necesidades vitales. 
En caso de las empresas esta asunción es difícil de mantener, pues muchas no necesitan terrenos donde se produzcan recursos bióticos. Un taller de reparación de coches, una entidad financiera, etc., realizan actividades sin ningún vínculo directo con este tipo de recursos. Surge entonces el concepto de contrahuella. El punto de partida es que, si bien es deseable que las empresas reduzcan su huella siendo más eficientes, esto es, reduciendo sus consumos, se considera positivo que dispongan de espacios naturales, permitiendo que inversiones en este tipo de superficie reduzcan la huella. De este modo, la huella ecológica fomentaría que el sector privado se involucre en la conservación de los espacios naturales (Doménech, 2007) lo que se considera positivo, en términos de sostenibilidad.

Las superficies de cultivos, pastos, bosques, jardines o, por ejemplo, reservas marinas, de que disponga la empresa contribuirán a contrarrestar una parte de la huella, pues son consideradas contrahuella. Para reducir la huella a una hectárea, bastaría con adquirir la misma cantidad de terreno de una de estas superficies. En el caso de que se invierta en superficie arbolada, se reducirán también las emisiones de $\mathrm{CO}_{2}$, considerando la tasa de absorción de $5,21 \mathrm{tCO}_{2} / \mathrm{ha} / \mathrm{año}$. Si a la HEC le restamos la contrahuella, obtenemos la HE neta, o de otro modo, la superficie que precisa la empresa menos que aquella de la que dispone.

Así, si en la HEC de los países es necesario disponer de una superficie ecológicamente productiva para no incurrir en déficit ecológico, en la huella de las organizaciones es positivo disponer de contrahuella, pues permite reducir su huella. Al igual que la superficie ecológicamente productiva, la contrahuella se multiplica, además que por el factor de emisión correspondiente, por un factor de rendimiento que pondera la productividad de la contrahuella en relación a los valores globales.

\section{Fortalezas y debilidades}

La metodología sigue, en lo fundamental, la filosofía del método desarrollado por Wackernagel y Rees. Es por tanto un método fácilmente asimilable para aquellos investigadores familiarizados con el análisis de huella desarrollado por estos dos autores. En cuanto a su alcance, hablamos de un método completo que permite añadir nuevos consumos a las categorías de propuestas, incorporando también la generación de residuos. Es, por tanto, un método flexible que permite su adaptación a las particularidades de cualquier tipo de organización. Igualmente, se expresa en hectáreas y toneladas de $\mathrm{CO}_{2}$, lo que incrementa su utilidad.

En cuanto a las debilidades, existe dificultad para obtener determinados factores de conversión (euros a hectáreas e intensidad energética, principalmente) precisos y actuales, pues las fuentes de información para su elaboración suelen ser escasas. Así mismo, se está trabajando en cuestiones como la incorporación del ciclo de vida de los combustibles consumidos, la incorporación de residuos peligrosos y gases diferentes del $\mathrm{CO}_{2}$, el uso de infraestructuras públicas por las organizaciones, tasas de absorción de pastos, cultivos y sistemas acuáticos, además de mejorar la precisión de la huella de los servicios y comidas de empresa. 


\section{CÁLCULO DE LA HUELLA ECOLÓGICA DE UNA EMPRESA PRODUCTORA DE MEJILLÓN EN GALICIA}

\subsection{El cultivo de mejillón en Galicia ${ }^{15}$}

El mejillón es un producto con un fuerte arraigo en Galicia y en la cultura gallega. Ya en los asentamientos celtas existen evidencias de su aprovechamiento por los humanos, quienes, aprovechando la baja mar, recogían uno de los moluscos más abundantes de las costas gallegas.

La primera batea ${ }^{16}$ de mejillón se instaló en Galicia en la Ría de Arousa en 1945 , aumentando de modo continuo la producción a partir de ese momento. En 1956 ya existían 249 bateas en Arousa, a las que se añaden otras 155 más distribuidas en las Rías de Viveiro, A Coruña, Noia, Pontevedra y Vigo.

En la actualidad, la producción de mejillón en Galicia se realiza en más de 3.300 bateas, situadas en las Rías de Vigo, Pontevedra, Arousa, Muros y Noia y Sada, generando la miticultura más de 7.000 empleos directos, a los que hay que añadir todos aquellos relacionados con su transformación y comercialización. La producción anual gallega se sitúa en la actualidad en torno a 250.000 , sólo superada por China.

En cuanto al cultivo, existen cuatro etapas principales:

- Obtención de la cría: el cultivo comienza con la recolección de mejillones pequeños (entre $0,25 \mathrm{~mm}$ y $2 \mathrm{~cm}$.), bien en las zonas rocosas o también mediante colectores artificiales que se colocan en las propias balsas de cultivo.

- Colocación de la cría en las cuerdas: la cría y, en su momento, el mejillón procedente del desdoble, se coloca a mano y con maquinaria especial al largo de las cuerdas de nailon, rodeándose de una red fina de algodón que sujeta al bivalvo hasta que produce unos filamentos denominados biso con los que se agarra a la cuerda. En cada cuerda se pueden colocar de 14 a 15 $\mathrm{kg}$ de cría y en cada $30-40 \mathrm{~cm}$ se insertan barras de plástico o madera (palillos) de 20 o $30 \mathrm{~cm}$ para evitar que cuándo la cría crezca se desprenda por su propio peso. Finalmente, las cuerdas se cuelgan de la batea empleando una cuerda más pequeña denominada rabiza.

- Desdoble o control del número de ejemplares: después de unos seis meses, la cría ocupa demasiado espacio como para poder permanecer sujeta en la cuerda sin desprenderse, procediendo a la fase de desdoble, que suele comenzar en verano, pudiendo prolongarse hasta bien entrado el otoño. En ella, el mejillón joven se coloca en otras cuerdas, donde dispone de espacio para seguir creciendo.

- Engorde y cosecha: el crecimiento de mejillón en las Rías gallegas es muy rápido, pudendo conseguir la talla comercial en un año. La temporada principal de cosecha es de octubre a marzo, cuando el mejillón está próximo a reproducirse y su peso es mayor. Sin embargo, el mejillón puede recogerse todo el año, ya que la producción se realiza de forma que se mantienen simultáneamente cuerdas de cría, engorde y cosecha.

El productor de mejillón (bateeiro), dispone de una embarcación especial para realizar las tareas de cultivo. El equipo con el que está dotada la embarcación puede variar notablemente, existiendo desde pequeños barcos de madera, sin más equipo con una grúa hidráulica, hasta modernos buques de casco de poliéster con 
una amplia gama de maquinaria a bordo (encordadora, desgranadora, etc.) que automatizan las tareas desempeñadas al largo del año. La tipología es tan diversa que existen bateeiros que realizan su labor simplemente con una pequeña lancha para desplazarse a la batea y una grúa fija, situada en el área central del entramado de vigas y puntales. Además de las propias tareas de cultivo, a lo largo del año se realizan diferentes trabajos de mantenimiento de la batea, tales como la reparación y relevo de puntales y vigas que habían podido estar dañadas, limpieza de las cuerdas, reparaciones de la embarcación y demás.

\subsection{Breve descripción de la empresa estudiada}

La empresa Alfa se dedica exclusivamente al cultivo de mejillón en la Ría de Arousa (Galicia), disponiendo para esta labor de dos bateas, con una producción anual total que varía entre 89.000 y 115.000 Kilos en los dos años estudiados, 2002 y 2007. Alfa cuenta con una embarcación que emplea para desplazarse hasta las bateas, recoger el mejillón y descargarlo en el puerto. Las labores a realizar se relacionan con el propio cultivo del bivalvo y el mantenimiento de las bateas y de la embarcación.

\subsection{Obtención de la información}

En la obtención de la información se distinguen dos fases. En primer lugar, se suministró información general de la $\mathrm{HE}$ a las personas que la empresa designó como responsables de la investigación, explicándoles los objetivos del estudio y el tipo de datos necesarios. Posteriormente, se elaboró un cuestionario que trataba de ser una guía, indicando las principales categorías de consumo necesarias para estimar la HEC. El cuestionario enviado no era cerrado, pudiéndose añadir las categorías de consumo que se consideraran oportunas. Tal y como señalamos, se solicitó información referida a los ejercicios económicos 2002 y 2007, de modo que es posible comparar la evolución de la HEC de las empresas estudiadas en un período de cinco años.

\subsection{Resultados}

Los principales resultados del estudio se resumen en las siguientes tablas que recogen la $\mathrm{HE}$, las emisiones de $\mathrm{CO}_{2}$, así como las principales ratios relacionadas con el análisis realizado. Se analiza la HEC en 2002, estudiando posteriormente los principales cambios en 2007.

\subsubsection{La HEC en 2002}

En el primero de los años estudiados, la empresa Alfa tiene una HEC bruta de 8,9 Gha. En la medida en que esta empresa no dispone de contrahuella, su HEC bruta coincide con la neta. La producción en ese año es de 116,6 t de mejillón, por lo que esta empresa precisa $0,08 \mathrm{Gha} / \mathrm{t}$ para producir una tonelada de mejillón. 
Tabla 3. Huella ecológica, emisiones de $\mathrm{CO}_{2}$ y ratios relacionados de la empresa Alfa

\begin{tabular}{|l|c|c|c|}
\hline Concepto & Unidad & $\mathbf{2 0 0 2}$ & $\mathbf{2 0 0 7}$ \\
\hline $\mathrm{HEC}$ bruta & $\mathrm{Gha}$ & 8,9 & 10,98 \\
\hline Contrahuella & $\mathrm{Gha}$ & 0,0 & 0,0 \\
\hline $\mathrm{HEC}$ neta & $\mathrm{Gha}$ & 8,9 & 11,0 \\
\hline Producción & $\mathrm{t}$ & 116,6 & 89,8 \\
\hline $\begin{array}{l}\text { Ratio HE neta/t } \\
\text { producción }\end{array}$ & $\mathrm{Gha} / \mathrm{t}$ & 0,08 & 0,12 \\
\hline $\begin{array}{l}\text { Emisiones } \mathrm{CO}_{2} \\
\text { brutas }\end{array}$ & $\mathrm{t}$ & 40,4 & 52,2 \\
\hline Emisiones $\mathrm{CO}_{2}$ netas & $\mathrm{t}$ & 40,4 & 52,2 \\
\hline $\begin{array}{l}\mathrm{t} \mathrm{CO}_{2} \text { netas/t } \\
\text { mercancía }\end{array}$ & $\mathrm{t}$ & 0,35 & 0,58 \\
\hline
\end{tabular}

Fuente: Elaboración propia

En cuanto a la distribución de la HEC entre las distintas superficies (Tabla 6), el $86,35 \%$ de la huella de Alfa en $2002,7,71$ Gha, procede de la energía incorporada, directa o indirectamente, a los distintos consumos. El resto de la HEC se origina en el consumo de productos relacionados con la superficie "bosques", $(0,05 \mathrm{Gha}$, el $0,35 \%$ de la HEC), "superficie construida" ( 0,01 Gha, y el $0,04 \%)$ y "mar" $(1,17$ Gha y el $13,07 \%$ de la HEC total).

Tabla 4. La HEC bruta de Alfa: tipos de superficies

\begin{tabular}{|c|c|c|c|c|}
\hline Tipo de superficie & $\begin{array}{l}\text { Gha } \\
2002\end{array}$ & $\%$ & $\begin{array}{l}\text { Gha } \\
2007\end{array}$ & $\%$ \\
\hline Energía fósil & 7,71 & $86,35 \%$ & 9,80 & $89,2 \%$ \\
\hline Superficie cultivable & 0,00 & $0,00 \%$ & 0,00 & $0,0 \%$ \\
\hline Pastos & 0,00 & $0,00 \%$ & 0,00 & $0,0 \%$ \\
\hline Bosque & 0,05 & $0,52 \%$ & 0,2 & $2,1 \%$ \\
\hline $\begin{array}{l}\text { Superficie } \\
\text { construida }\end{array}$ & 0,01 & $0,06 \%$ & 0,01 & $0,1 \%$ \\
\hline Mar & 1,17 & $13,07 \%$ & 0,95 & $8,7 \%$ \\
\hline Total & 8,93 & $100 \%$ & 10,98 & $100 \%$ \\
\hline
\end{tabular}

Fuente: Elaboración propia

La HEC relacionada con la energía fósil surge, principalmente, por el consumo de combustible de la embarcación de la que dispone Alfa. Este concepto supone una HEC de 5,98 Gha, el $77,8 \%$ de la HEC de la energía. El resto de la huella energética se genera en los consumos de diferentes materiales, precisos para realizar la actividad y mantener en buen estado de conservación las bateas (ropa de trabajo, aceites, productos de limpieza, cuerdas y rabizas, red para fijar la cría, etc.), además de la amortización del barco y de las dos bateas con las que cuenta la empresa. 
La energía incorporada en todos estos conceptos supone 1,34 Gha, que sumadas a las 5,98 Gha procedentes del consumo de combustibles alcanzan 7,32 Gha, casi la totalidad de la HEC energética. Las restantes $0,33 \mathrm{Gha}$, tienen que ver con la energía necesaria para realizar algunos servicios, principalmente de reparación y mantenimiento de la embarcación y con el gasto energético realizado en el abastecimiento de cría.

En cuanto al resto de componentes, es necesario destacar la huella asociada al consumo de productos del mar, derivada de la compra de cría de mejillón $(0,27$ Gha) y la ocupación de la superficie marítima realizada por las bateas (0,90 Gha). En este último caso, se computa no sólo la superficie ocupada por una, sino el total de superficie del polígono de bateas que le corresponde, estimada en una hectárea por vivero.

En relación a la superficie construida, se recoge el uso de un galpón, empleado como almacén, que no supuso coste alguno para Alfa, por lo que no computa en la matriz de obras. La HEC de productos que proceden de los bosques se corresponde al consumo de palillos, empleados para evitar que la cría se desprenda de las cuerdas cuando aumenta de tamaño.

Tabla 5. La HEC de Alfa: emisiones brutas de $\mathrm{CO}_{2}$

\begin{tabular}{|c|c|c|c|c|}
\hline Tipo de superficie & $\begin{array}{l}\mathrm{tCO}_{2} \\
2002\end{array}$ & $\%$ & $\begin{array}{l}\mathrm{tCO}_{2} \\
2007\end{array}$ & $\%$ \\
\hline Energía fósil & 40,13 & $99,40 \%$ & 51,0 & $97,75 \%$ \\
\hline Superficie cultivable & 0,00 & $0,00 \%$ & 0,0 & $0,00 \%$ \\
\hline Pastos & 0,00 & $0,00 \%$ & 0,0 & $0,00 \%$ \\
\hline Bosque & 0,24 & $0,60 \%$ & 1,2 & $2,25 \%$ \\
\hline $\begin{array}{l}\text { Superficie } \\
\text { construida }\end{array}$ & 0,00 & $0,00 \%$ & 0,0 & $0,00 \%$ \\
\hline Mar & 0,00 & $0,00 \%$ & 0,0 & $0,00 \%$ \\
\hline Total & 40,4 & $100 \%$ & 52,20 & $100,0 \%$ \\
\hline
\end{tabular}

Fuente: Elaboración propia

En cuanto a las emisiones de $\mathrm{CO}_{2}$ en 2002, ascienden a 40,4 toneladas, lo que significa que la obtención de una tonelada de mejillón produce 0,35 toneladas de este gas. Prácticamente el $100 \%$ de las emisiones, 40,13 toneladas de $\mathrm{CO}_{2}$, proceden de la energía incorporada a los diferentes consumos, mientras que sólo 0,24 toneladas se deben al consumo de productos de madera, cuya existencia implica la desaparición de superficie forestal, con capacidad para absorber $\mathrm{CO}_{2}$.

\subsubsection{La HEC en 2007}

La HEC bruta de Alfa en el año 2007 aumenta un 23,3\%, alcanzando 10,98 Gha (Tabla 5). En la medida en que sigue sin existir contrahuella, la HEC neta alcanza el mismo valor. Este aumento es incluso más acentuado si relacionamos el indicador con la producción, que cae de 116,6 t en 2002 a 89,8 en 2007. De este modo, en el último año estudiado, se necesitan 0,12 Gha para producir una tonelada de mejillón, lo que significa un aumento del $50 \%$ respeto al primero año estudiado. 
Los motivos de este aumento tienen que ver, en lo fundamental, con el incremento de la huella de la energía fósil (Tabla 6) que crece un 27,1\%, suponiendo 9,80 Gha. Este incremento se explica, en buena medida, por un mayor consumo de materiales, cuya huella consigue ahora las $3,99 \mathrm{Gha}$, frente las sólo 1,34 Gha en 2002. Un mayor consumo de pintura, 2,7 Gha en 2007 , frente a sólo $0,14 \mathrm{GHa}$ en 2002, propicia una mayor HEC de los materiales. Igualmente, surge una HEC de 0,74 Gha, derivada del consumo de energía, preciso para el reciclaje del poliéster, generado como consecuencia del desarme de una batea, sustituida en este año.

El resto de componentes siguen teniendo escasa relevancia. La HEC de los productos del mar se mantiene como el segundo componente más importante, a pesar de un descenso de 0,22 Gha debido a un menor consumo de cría de mejillón. En 2002, año en el que Alfa comenzó su actividad, se compraron 3,38 toneladas de mejilla ${ }^{17}$, la necesaria para poner en marcha la producción de las dos bateas de Alfa. En 2007, con el proceso productivo ya en funcionamiento, sólo fue necesario adquirir 0,6 toneladas, cantidad suficiente para cubrir las cuerdas vacías en ese año.

La huella de los productos asociados a la superficie "bosques" se multiplica por cuatro, debido a la renovación de vigas de las bateas, de madera de eucalipto, aunque en términos absolutos sólo supone 0,2 Gha. La superficie construida no varió en el período estudiado.

\section{CONSIDERACIONES FINALES}

En esta investigación empleamos el método denominado MC3 para calcular la HEC de una empresa productora de mejillón en Galicia en dos años diferentes, 2002 y 2007, expresándola en hectáreas estandarizadas (Gha) y toneladas de $\mathrm{CO}_{2}$. Nuestro trabajo trata de detectar aquellas áreas que generan una mayor huella, además de estudiar la evolución del indicador en el período considerado. De este modo, se pretende ofrecer información relevante para la gestión medioambiental de la empresa.

Los resultados obtenidos muestran una HE no muy elevada, asociada principalmente al consumo de energía. Debemos destacar que el cultivo de mejillón es una actividad realizada al aire libre, que no precisa del empleo de alimentos artificiales, ni medicamentos empleados en otro tipo de acuicultura. La labor de los productores consiste, "simplemente", en hacer crecer de modo natural un producto que adquieren en las primeras fases de su ciclo de vida. Pese al desarrollo experimentado en los últimos años, no es un proceso que necesite una gran cantidad de inputs, más que los necesarios para el desplazamiento del productor a las bateas, y el manejo y transporte del producto. Sin embargo, los rendimientos por hectárea y la producción son elevados, lo que favorece que la HEC se reduzca al relacionarla con la cantidad de producto obtenido.

Si profundizamos en el análisis de los elementos que generan huella, observamos cómo un único consumo, el combustible empleado por los motores de la embarcación de la empresa, supone más de $2 / 3$ de la huella correspondiente al consumo de energía y más del $50 \%$ de la huella total de Alfa. De ahí que las acciones encaminadas a reducir la HEC de esta empresa deban prestar especial atención a la búsqueda de una mayor eficiencia en este campo.

Otros elementos relevantes en cuanto a la generación de HEC se relacionan con el consumo de determinados materiales (ropa de trabajo, aceites, cuerdas, productos 
de limpieza...) que, indirectamente, contribuyen a la huella de la energía. Completando los principales generadores de HEC, estaría el consumo de cría de mejillón, además de la superficie ocupada por las bateas.

En lo que se refiere a la evolución del indicador en el período considerado, se observa un incremento destacable, más de un $23 \%$, provocado por un mayor consumo de energía. No se trata tanto un incremento del consumo directo de energía, sino de un aumento del consumo de determinados materiales, pintura principalmente, además de surgir huella derivada del reciclaje de una parte de la batea, que se reemplaza por otra nueva. Alfa no debe descuidar la gestión de los materiales, pero debemos tener en cuenta que el reciclaje del poliéster se producirá sólo en el momento que dé la sustitución de la batea, por lo tanto, de modo muy esporádico, si bien se trata de una parte de la huella que, cuando surge, es inevitable. De modo análogo, cada cierto periodo se deben realizar tareas de mantenimiento general que pueden suponer un mayor consumo de pintura.

\section{REFERENCIAS BIBLIOGRÁFICAS}

- Bicknell, K.B., Ball, R.J., Cullen, R., Bigsby, H.R., (1998): "New methodology for the ecological footprint with an application to the New Zealand economy". Ecological Economics. Vol. 27. pp 149-160.

- Carballo Penela, A., García Negro, M.C. (2009) "La huella ecológica de una explotación mejillonera". Revista Luna Azul (en revisión).

- Doménech, J.L. (2004a): "Huella ecológica portuaria y desarrollo sostenible". Puertos. Vol.114. pp. 26-31

- Doménech, J.L. (2004b): "La huella ecológica empresarial: el caso del puerto de Gijón". Actas del VII Congreso Nacional de Medio Ambiente. 22-26 Nov., 2004. Madrid.

- Doménech, J.L., (2007): Huella ecológica y desarrollo sostenible. AENOR Ediciones, Madrid.

- Ibañez Etxeburúa, N., (2001): La huella ecológica de Donostia-San Sebastián.http://lunazul.ucaldas.edu.co/= (último acceso, septiembre, 2006).

- IPCC, International Panel on Climate Change, (2001): IPCC Working Group I: The scientific basis (http://www.grida.no/CLIMATE/IPCC_TAR/wg1/index.htm; último acceso, junio, 2008).

- Lenzen, M., Lundie, S., Bransgrove, G., Charet, L., Sack, F., (2003): "Assessing the Ecological Footprint of a Large Metropolitan Water Supplier: Lessons for Water Management and Planning towards Sustainability". Journal of Environmental Planning and Management. Vol. 46, pp. 113-141.

- Lenzen, M., Murray, S., (2001): "A modified ecological footprint method and its application to Australia". Ecological Economics. Vol. 37, pp. 229-255.

- Mayor Farguell, X., Quintana Gozalo, V., Belmonte Zamora, R., (2003): Aproximación a la huella ecológica de Cataluña. http://www.catsostenible.org/pdf/DdR_7_Huella_Ecologica.pdf(último acceso, junio, 2006).

- McDonald, G. and Patterson M., (2003): Ecological Footprints of New Zealand and its Regions. New Zealand: Ministry for the Environment. (http://www.mfe.govt.nz/publications/ser/eco-footprint-sep03/index.html; último acceso, enero, 2007). 
- Monfreda, Ch., Wackernagel, M., Deumling, D. (2004): "Establishing national natural capital accounts based on detailed Ecological Footprint and biological capacity assessment". Land use Policy. Vol. 21, pp. 231-246.

- Rees, W.E., (2000): "Eco-footprint analysis: merits and brickbats". Ecological Economics. Vol. 32, pp. 371-374.

- Relea Ginés, F., Prat Noguer, A., (1998): Aproximación a la huella ecológica de Barcelona. Barcelona: Ajuntament de Barcelona. (http://www.mediambient.bcn.es/cas/down/masu6_1.pdf (Último acceso, noviembre 2005).

- Simmons C., Chambers N., (1998): "Footprinting UK Households: "How big is your ecological garden?" Local Environment Vol.3, No.3

- Simmons, Lewis and Barrett., (2000): "Two feet-two approache s: a component-based model of ecological footprinting," Ecological Economics, vol.32, pp. 75-380, 2000.

- Wackernagel, M., (1999): "An evaluation of the ecological footprint". Ecological Economics. Vol. 31, pp. 317-318.

- Wackernagel, M., Dholakia, R., Deumling, D., Richardson, D., (2000): Redefining Progress, Assess your Household's Ecological Footprint 2.0, March 2000. http://greatchange.org/ng-footprint-ef_household_evaluation.xls (último acceso, noviembre, 2005).

- Wackernagel, M. Monfreda, C. (2004): "Ecological footprints and energy". Encyclopedia of energy 2, pp. 1-11.

- Wackernagel M., Onisto L., Bello P., Callejas Linares, A., López Falfán, I. S., Méndez García, J., Suárez Guerrero, A. I., Suárez Guerrero, M. G., (1999): "National natural capital accounting with the ecological footprint concept". Ecological Economics. Vol. 29, pp. 375-90.

- Wackernagel, M., Rees W. E., (1996): Our Ecological Footprint: Reducing Human Impact on the Earth, New Society PublisHE rs, Philadelphia.

- Wackernagel, M., Silverstein, J., (2000): "Big things first: focusing on the scale imperative with the ecological footprint". Ecological Economics. Vol. 32, pp. 391-394.

- Wiedmann T., Barret, J., Lenzen M. (2007): "Companies on the Scale: Comparing and Benchmarking the Footprints of Businesses". International Ecological Footprint Conference, 8-10 Mayo 2007, Cardiff (UK). (http://www.brass.cf.ac.uk/uploads/Wiedmann_et_al_P36.pdf; último acceso, septiembre, 2007)

- Wiedmann, T. and Lenzen, M., (2006): "Sharing Responsibility along Supply Chains - A New Life-Cycle Approach and Software Tool for Triple-Bottom-Line Accounting". Corporate Responsibility Research Conference 2006, 4-5 September 2006, Trinity College Dublin, Ireland.

- Wiedmann, T., Minx, J. (2007): A definition of carbon footprint. Durham: ISA UK Research Report 07-01: (http://www.isa-research.co.uk/docs/ISAUK_Report_07-01_carbon_footprint.pdf (ultimo acceso, abril, 2008)

- Wiedmann, T., Minx, J., Barret, J., Wackernagel, M., (2006): "Allocating ecological footprints to final consumption categories with input-output analysis". Ecological Economics. Vol. 58, pp. 28-48.

\section{NOTAS}

1. Wackernagel y Rees (1996, 51-52); Rees (2000). La forma de la definición se ha modificado, precisando algunas cuestiones. En la actualidad, Global Footprint Network (GFN), organismo en el que participa Wackernagel y que, entre otras cosas, trata de avanzar en la estandarización de la metodología de cálculo y en la 
difusión del indicador, define la huella ecológica como: "una medida de cuánta superficie biológicamente productiva, incluyendo agua y tierra, necesita un individuo, población o actividad para producir todos los recursos que consume y absorber los deshechos que genera, empleando la tecnología y las prácticas de gestión existentes". De este modo, se manifiesta explícitamente en la definición la posibilidad de aplicar la huella la realidades diferentes de poblaciones,

2. Si la HE es menor que la superficie disponible, existiría una reserva o superávit ecológico.

3. En la actualidad existen otras alternativas metodológicas para estimar la HE de países y regiones. La aproximación de los componentes (Simmons y Chambers, 1998; Simmons et al., 2000) o el cálculo de la HE empleando técnicas input-output (Bicknell et al. 1998; Lenzen y Murray 2001; McDonald y Patterson, 2003; o Wiedmann et al., 2006) son las que han conseguido mayor difusión.

4. Esta sería una explicación general del método de cálculo. No obstante, se han ido añadiendo más elementos que los que hemos mencionado, como factores de equivalencia y rendimiento, dando lugar al cálculo de la HE expresado en hectáreas estandarizadas (Gha); diferenciación entre productos primarios y secundarios.

5. De ahí que autores como Mayor et al., (2003) consideren la posibilidad de incrementar la huella considerando una superficie de 282.743 hectáreas, el área de exclusión establecida tras el accidente de Chernobyl en 1986.

6. Estas superficies son incluidas frecuentemente en la sección de la superficie construida, y no en la huella de la energía.

7. Si bien este ajuste es coherente con los objetivos de la huella, orientados a estimar el impacto de los habitantes de un territorio, lo cierto es que puede fomentar comportamientos no deseados. Tanto Ibáñez (2001) como Mayor (2003) señalan que podría darse la paradoja de que un país intente reducir su huella exportando bienes intensivos en el consumo de energía, aun soportando los efectos medioambientales de la producción de energía. Igualmente, la huella sería menor sí se importan bienes con un contenido energético más bajo.

8. En Wiedmann y Minx (2007) se puede encontrar abundante información sobre la huella del carbono.

9. Al igual que en la estimación de la HE de habitantes de territorios, la "aproximación de los componentes" y las análisis input-output (Wiedmann et al., 2007; Wiedmann y Lenzen, 2006) se emplean igualmente para el cálculo de la HE corporativa.

10. El cálculo de las hectáreas estandarizadas se realiza empleando factores de equivalencia y rendimiento, del mismo modo que en la versión tradicional del indicador.

11. Los subgrupos son electricidad, combustibles, materiales, materiales de construcción, servicios y deshechos. 
12. Estos factores de intensidad energética serían del mismo tipo que los empleados en el cálculo de la huella de países, para determinar la cantidad de energía incorporada a los flujos comerciales.

13. Por ejemplo, los combustibles líquidos tienen, en términos medios, un factor de emisión de carbono de $0,0734 \mathrm{tCO}_{2} / \mathrm{Gj}$. Si consideramos la tasa de absorción de $5,21 \mathrm{tCO}_{2} / \mathrm{ha} / \mathrm{año}$, una hectárea de bosque podría absorber $70,98 \mathrm{Gj}$ de combustibles líquidos al año $(5,21 / 0,0734)$, valor que se tomaría como productividad energética de los combustibles líquidos.

14. Así, por ejemplo, si tenemos un consumo de 10 toneladas de uvas y la productividad de las uvas es de 9,3 t/ha., la huella de las uvas sería de 1,07 ha, asignada a la superficie cultivos, al igual que se haría en la huella de un país o región.

15. No que atangue ás fases de cultivo, seguimos o exposto por Antonio Figueras en: http://weblogs.madrimasd.org/ciencia_marina/archive/2008/07/10/68192.aspx

16. Las bateas son artefactos flotantes de madera que sostienen las cuerdas a las que se adhiere el mejillón.

17. Cría de mejillón 